\title{
Rufausnutzung zwischen Lauterkeitsrecht und Markenschutz: Umwege und Auswege
}

Im Wettbewerb zwischen Unternehmen kommt es immer wieder vor, dass sich Produzenten insbesondere bei der Produktbezeichnung an bereits etablierte Bezeichnungen von Fabrikaten ihrer Mitbewerber anlehnen, um Assoziationsvorteile zu generieren. Entsprechende Sachverhalte werfen Fragen sowohl des Lauterkeits- wie auch des Immaterialgüterrechts auf. Ihre sachgerechte Beantwortung sollte sich nicht nur auf technische Fragen des Vor-bzw. Nachrangs der jeweils angerufenen Abwehrnormen beschränken, sondern muss der Grundsatzkonzeption der beiden Rechtsgebiete und ihrem Zusammenspiel gebührend Rechnung tragen. Ein aktueller Entscheid des Handelsgerichts Bern gibt Anlass dazu.

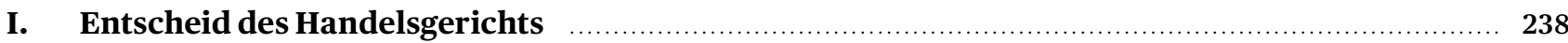

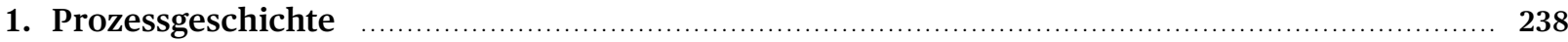

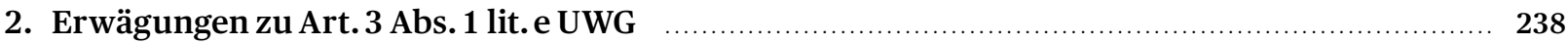

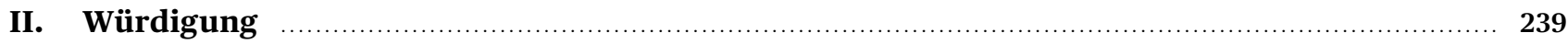

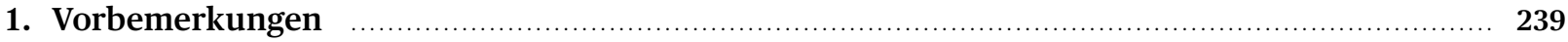

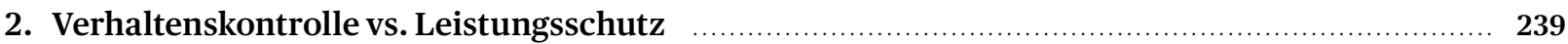

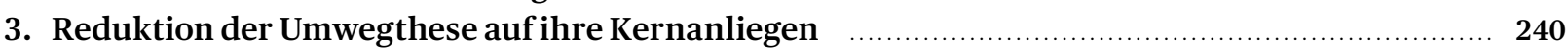

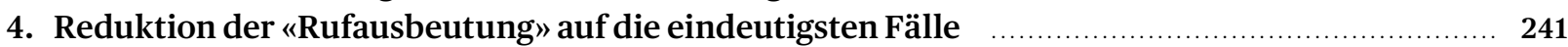

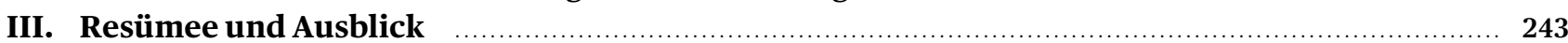

Zitiervorschlag:

RETO HEIZMANN / LEANDER D. LOACKER, Rufausnutzung zwischen Lauterkeitsrecht und Markenschutz, sui generis 2021, S. 237

PDDr. iur. Reto Heizmann, Privatdozent an der Universität Zürich, Präsident des Kantonsgerichts Schwyz.

Prof.Dr.iur. Leander D. Loacker, M.Phil., Professor an der Universität Zürich, Of Counsel in Zürich.

URL: sui-generis.ch $/ 187$

DOI: https://doi.org/10.21257/sg.187

Dieses Werk ist lizenziert unter einer Creative Commons Namens-

nennung - Weitergabe unter gleichen Bedingungen 4.0 International

Lizenz. 


\section{Entscheid des Handelsgerichts}

\section{Prozessgeschichte}

1 Am 24.Juli 2019 reichte die Peppe GmbH beim Handelsgericht des Kantons Bern Klage gegen die MaFuMa GmbH ein und beantragte, es sei der Beklagten zu verbieten, unter der Bezeichnung «Summerer» einen Ingwer-Likör anzubieten, in Verkehr zu bringen oder zu diesem Zweck zu lagern. ${ }^{1}$

2 Der Ingwer-Likör «Ingwerer» ist das Hauptprodukt der Klägerin (E. 11.1). ${ }^{2}$ Sie warf der Beklagten unlauteres Verhalten nach Art. 3 Abs. 1 lit. e UWG ${ }^{3}$ vor, weil diese sich mit deren Produkt «Summerer» unnötig an die Produkte der Klägerin angelehnt habe (E.1). Der «Summerer» enthält u.a. Zitronen und Ingwer (E.11.2). Während die Unternehmensgeschichte der Klägerin auf das Jahr 2013 zurückreicht, wurde die Beklagte erst im Jahr 2019 gegründet (E. 12 ff.). Die Klägerin lancierte im Jahr 2017 überdies den Gin-Ingwer-Likör «Gingwerer» und präsentierte im Jahr 2018 zum Meistertitel des Fussballclubs «YB» den «Ingwerer» mit der Etikette «Meisterer» sowie im Jahr 2019, nach der Titelverteidigung, mit der Etikette «Meistererer» (E. $16 \mathrm{ff}$.$) . Im Sommer 2019$ veranstaltete sie für Mitarbeiter, Freelancer und Bekannte den Sommeranlass «Sommerer» (E. 20). ${ }^{4}$ Die Beklagte präsentierte ihren «Summerer» erst anlässlich der Eröffnungsparty vom 30. Mai 2019 (E. 21).

3 Nach den üblichen Verfahrensschritten im Direktprozess (E. 7) ${ }^{5}$ entschied das Handelsgericht am 24. Februar 2020 (Ausfertigung am 9.Juni 2020), der Beklagten werde «ab 30 Tagen seit Eröffnung des begründeten Entscheids verboten, unter der Bezeichnung «Summerer einen Ingwer-Likör anzubieten, in Verkehr zu bringen oder zu diesem Zweck zu lagern» (Dispositivziffer 1; E. 31). Dieser Entscheid wurde rechtskräftig, ohne Weiterzug an das Bundesgericht. ${ }^{6}$

\section{Erwägungen zu Art. 3 Abs. 1 lit.e UWG}

4 Wie eingangs erwähnt, stützte die Klägerin ihre Klage auf Art. 3 Abs. 1 lit. e UWG, wonach derjenige unlauter handelt, der sich, seine Waren, Werke, Leistungen oder deren

1 Urteil des Handelsgerichts des Kantons Bern HG19 89 vom 24. Feb ruar 2020 E. 1 (unter Kosten- und Entschädigungsfolge) = iusNet IP vom 18. Dezember 2020.

2 Sie ist Eigentümerin der Schweizer Marke Nr. 660’012 «INGWERER» (E.11.1).

3 Bundesgesetz gegen den unlauteren Wettbewerb vom 19. Dezember 1986 (UWG; SR 241).

4 Die Einladung erfolgte durch einen «newsletterer» (E. 20).

5 Art. 5 Abs. 1 lit. d ZPO (Schweizerische Zivilprozessordnung vom 19. Dezember 2008 [ZPO; SR 272]), der Streitwert lag bei CHF100'000 (E. 33.2).

6 Vgl. auch Der Bund vom 22.Juli 2020 («Ingwerer-Konkurrentin ist konkurs»).
Preise in unrichtiger, irreführender, unnötig herabsetzender oder anlehnender Weise mit anderen, ihren Waren, Werken, Leistungen oder deren Preisen vergleicht oder in entsprechender Weise Dritte im Wettbewerb begünstigt. Der Entscheid handelt von der Tatbestandsvariante der unnötigen Anlehnung bzw. der sog. Rufausbeutung.

Das Handelsgericht erklärte unter Bezugnahme auf die 5 Bundesgerichtspraxis, zu prüfen sei der Ruf der Waren der Klägerin und allenfalls deren Verkehrsdurchsetzung oder Bekanntheit, das Vorliegen eines Image-Transfers, indem mit dem Marktauftritt Gedankenassoziationen zu den Waren der Klägerin geweckt würden, und schliesslich, ob die Anlehnung unnötig erfolgt, mithin sachlich nicht gerechtfertigt sei (E. 26).

Das Handelsgericht erläuterte, die Klägerin habe «nicht 6 eine eigentliche Verkehrsdurchsetzung, sondern höchstens deren Bekanntheit» nachzuweisen, wobei die Frage letztlich offenbleiben konnte, weil der Klägerin nach Auffassung des Handelsgerichts der Beweis der Bekanntheit ohnehin gelang (E. 27.4). Geführt wurde dieser Nachweis insbesondere unter Berufung auf die konstante Berichterstattung in den Medien und den stetig steigenden Umsatz (E. 27.5 f.). Das Handelsgericht bejahte sowohl den guten Ruf als auch die Bekanntheit der Produkte der Klägerin (E. 27.8).

Im Weiteren prüfte das Handelsgericht das Tatbestands- 7 merkmal der Rufausbeutung i.e.S., also das Vorliegen eines Image-Transfers (E. 28). Allgemein hielt es fest, dass die Rufausbeutung unabhängig von der Gefahr allfälliger Verwechslungen als unlauter gelte (E. 28.1). Die Klägerin brachte vor, sie habe mit dem Kennzeichen gespielt und neben dem «Ingwerer» einen «Gingwerer» und einen «Meisterer» auf den Markt gebracht (E. 28.2). Der «Summerer» werde in die Produktefamilie der Klägerin eingeordnet (E. 28.2). Das Handelsgericht folgte dieser Argumentation und kam zum Schluss, die Bezeichnung eines Zitronen-Ingwer-Likörs als «Summerer» sei objektiv geeignet, als weiteres Produkt der Klägerin wahrgenommen zu werden (E. 28.7).

Art. 3 Abs. 1 lit. e UWG setzt schliesslich voraus, dass die 8 Anlehnung bzw. Rufausbeutung «unnötig» ist. Nach Auffassung des Handelsgerichts informiere die Bezeichnung «Summerer» nicht über den Inhalt des Getränks; entgegen der Ansicht der Beklagten sei mit «Essenz des Sommers» nicht klar, woraus das Produkt bestehe (E. 29.3). Eine Förderung der Markttransparenz sei mit der Bezeichnung «Summerer» nicht verbunden (E. 29.3).

Für die Verwendung der Bezeichnung bestand nach An- 9 sicht des Handelsgerichts ebenso wenig ein Rechtfertigungsgrund: Die Beklagte habe erklärt, «Summerer» sei 
eine Kombination von «summer» als englisches Wort für Sommer und der ersten Wortsilbe von «Erfrischungsgetränk», der Name «Summerer» sei berndeutsch und die Steigerungsform «er» allen bekannt, und schliesslich sei die Endung «er» im berndeutschen Dialekt eine Steigerungsform, der Vertreter der Beklagten kenne vom Grossvater den Obstler (E. 29.4). Keine dieser Erklärungen konnte das Handelsgericht überzeugen, weshalb nach seiner Ansicht die Bezeichnung «Summerer» nicht durch legitime Informationsbedürfnisse gerechtfertigt war und die Anlehnung somit unnötig erfolgte(E. 29.4f.).

10 Die Frage, ob die in der Lehre vertretene Auffassung, eine Anlehnung durch Rufausbeutung sei stets unnötig, weil ein Aufklärungs- oder Informationsinteresse des Publikums nicht bestehe und es selten erforderlich sein dürfte, den konkret mit einem bestimmten Marktteilnehmer verbundenen Appeal-Faktor für die eigene Werbung auszunutzen, liess das Handelsgericht offen, weil nach seiner Auffassung ohnehin keine sachliche Rechtfertigung für die Anlehnung bestand (E. 29.2).

11 Damit waren nach Überzeugung des Handelsgerichts alle Tatbestandsvoraussetzung von Art. 3 Abs. 1 lit. e UWG erfüllt und das Anbieten, Inverkehrbringen und die Lagerung des Likörs «Summerer» unzulässig (E. 30).

\section{Würdigung}

\section{Vorbemerkungen}

12 In Bezug auf die Anwendungsvoraussetzungen der in der Praxis und Lehre anerkannten «Rufausbeutung» bewegt sich das Urteil auf sicher etabliertem Terrain; weniger überzeugend ist das erzielte Ergebnis, und zwar insbesondere mit Blick auf vertiefungswürdige Abgrenzungen des Verhältnisses zwischen Lauterkeits- und Immaterialgüterrecht. Dementsprechend sollen hier zunächst Ausführungen zum Verhältnis zwischen der lauterkeitsrechtlichen Verhaltenskontrolle und dem immaterialgüterrechtlichen Leistungsschutz (2.) erfolgen, welche sodann überleiten zur Frage der Anwendung der Umwegthese (3.). Den Schluss bildet eine knappe Darstellung der Implikationen in Bezug auf das lauterkeitsrechtliche Konzept der «Rufausbeutung» (4.).

\section{Verhaltenskontrolle vs. Leistungsschutz}

Gemeinhin gilt, dass das Lauterkeitsrecht Verhalten kontrolliert und das Immaterialgüterrecht Leistungen ${ }^{7}$ schützt. In den Worten des Bundesgerichts: «Das UWG

7 Diese Feststellung ist insofern verallgemeinernd, als die Marke als solche genau besehen nicht als Leistung geschützt wird, JOCHEN GLÖCKNER, Markenschutz durch Art. 3 lit. e UWG, sic! 2011, S. 274. bezweckt die Gewährleistung der Lauterkeit des Wettbewerbs, während es dem Immaterialgüterrecht vorbehalten ist, besondere Leistungen als solche zu schützen. Leistungen sind daher durch das UWG nicht als solche, sondern nur bei Vorliegen lauterkeitsrechtlich relevanter Umstände gegen Übernahme und Nachahmung geschützt, wie namentlich bei vermeidbarer Herkunftstäuschung, Rufausbeutung, hinterlistigem Verhalten oder behinderndem systematischem Vorgehen».8 Nach gängiger Auffassung schützt das schweizerische UWG Leistungen weder unmittelbar noch mittelbar. ${ }^{9}$

Dementsprechend sind Immaterialgütergesetze und 14 das UWG grundsätzlich losgelöst voneinander anzuwenden: So ist etwa der lauterkeitsrechtliche Schutz von Kennzeichen unabhängig von markenrechtlichen Eintragungserfordernissen zu beurteilen, womit ggf. zwar der Markenschutz nicht greift, wegen Schaffung von Verwechslungsgefahr u.dgl. aber das UWG eine Handhabe gegen entsprechendes Verhalten bieten kann. ${ }^{10}$ In konsequenter Umsetzung des Selbstständigkeitspostulats führt ebenso ein Verzicht auf Sonderrechtsschutz weder zu Nachteilen noch zu Vorteilen bezüglich der Anwendung des UWG.11

Diese Unterscheidung bzw. Aufgabenteilung ist jedoch 15 nur dogmatisch scharf zu ziehen: Zwar kann vorliegend nur gemutmasst werden, weshalb die Klägerin ihre Marke (E. 11.1) ${ }^{12}$ und deren Sperrkompeten $z^{13}$ nicht gerichtlich durchsetzen wollte oder konnte (E. 28.7). ${ }^{14}$ Grundsätzlich möglich wäre dies wohl vor demselben Gericht gewesen. ${ }^{15}$ Ein Beweggrund für die Wahl des lauterkeitsrechtlichen Schutzinstrumentariums gegen Rufausbeutung mag freilich gewesen sein, dass der Nachweis einer Verwechslungsgefahr ${ }^{16}$ nur bei einem Vorgehen nach

8 Urteil des Bundesgerichts 4A 86/2009 vom 26. Mai 2009 E.4.1 i.f. (nicht abgedruckt in BGE135 III 446); vgl. auch BGE131 III 384 E. 5.1.

9 ANDREAS HEINEMANN, in: Heizmann/Loacker (Hrsg.), UWG, Kommentar, Zürich 2018, §5 N 36, vgl. auch N 41 (zit. Heizmann/LoackerBEARBEITERIN); vgl. aber CARL BAUDENBACHER, in: Baudenbacher (Hrsg.), Lauterkeitsrecht, Kommentar zum Gesetz gegen den unlauteren Wettbewerb (UWG), Basel 2001, Art. 2 UWG N197.

10 Heizmann/Loacker-HEINEMANN, § 5 N 42.

11 Heizmann/Loacker-HEINEMANN, §5 N 44 .

12 Schweizer Marke Nr.660’012 «Ingwerer».

13 EUGEN MARBACH / PATRICK DUCREY / GREGOR WILD, Immaterialgüter- und Wettbewerbsrecht, 4. Aufl., Bern 2017, N743.

14 Die Beklagte behauptete, die Marke habe ihren Schutz verloren, was im Entscheid jedoch ungeklärt blieb.

15 Vgl. Art. 5 Abs. 1 lit. a ZPO für die sachliche Zuständigkeit.

16 Das Ausschliesslichkeitsrecht des Markeninhabers wird nicht verletzt, wenn Marken zweifelsfrei auseinander gehalten werden können; bei blosser Ausnutzung, nicht aber Benutzung der Marke, mit Ausnahme der Verwendung «berühmter Marken» (Art.15 MSchG), greift das MSchG nicht, CHRISTIAN SCHMID, in: Hilty/Arpagaus (Hrsg.), Basler Kommentar, Bundesgesetz gegen den unlauteren Wettbewerb, Basel 2013 (zit. BSK-BEARBEITERIN), Art. 3 Abs. 1 lit. e UWG N103, m.N.; vgl. auch die Nachweise in sogleich folgender Fn. 17. 
dem Markenschutzgesetz, nicht aber nach Art. 3 Abs. 1 lit. e UWG erforderlich ist (E. 25). ${ }^{17}$ Das MSchG18 erfasst eine blosse Kennzeichenanlehnung ausserhalb berühmter Marken nämlich nicht. ${ }^{19}$ Zumindest im Ergebnis erreichte die Klägerin über das lauterkeitsrechtliche Verbot der Rufausbeutung ohnehin dasselbe, was sie ggf. über das Markenschutzgesetz hätte durchsetzen können, nämlich das gerichtliche Verbot des Anbietens, Inverkehrbringens und der Lagerung des Likörs «Summerer» (vgl. Art. 55 ff. MSchG). ${ }^{20}$ So kann das Konzept der Rufausbeutung defacto zu einem Leistungsschutz führen, der durch die Immaterialgüterrechte in den betreffenden Fällen wohl häufig nicht zu erreichen wäre. Damit büsst das konzeptionelle Dogma, wonach das Lauterkeitsrecht nur Schutz vor unlauterem Verhalten biete, nicht aber Leistungen als solche schütze, jedenfalls bei rein ergebnisorientierter Betrachtung des konkreten Rechtsverhältnisses seine praktische Bedeutung weitgehend ein.

16 Generell stellt sich damit die Frage, wie weit das Lauterkeitsrecht Leistungen unter der Schwelle des Schutzniveaus des Immaterialgüterrechts (faktisch) schützen soll. Die Relation zwischen einem solchen verdeckten lauterkeitsrechtlichen Leistungsschutz und dem Schutz durch das Immaterialgüterrecht gilt zu Recht als «eines der Hauptprobleme des Lauterkeitsrechts».21 Es ist hier nicht der Ort, das Verhältnis zwischen Immaterialgüterschutz und Wettbewerbsrecht aufzurollen.22 Gezeigt werden soll aber doch, dass die vom Bundesgericht früher vertretene, in der Literatur kritisierte und mittlerweile als grundsätzlich überholt geltende sog. Umwegthese immerhin die Gefahr eines überbordenden lauterkeitsrechtlichen Leistungsschutzes durchaus wirksam eindämmen konnte. Wie nachfolgend gezeigt wird, ist diese Umwegthese allerdings auf deren Kerngehalt zu beschränken.

17 BGE135III 446 E. 7.1; Heizmann/Loacker-STAUBER/ISKIC, Art.3 Abs.1 lit. e N 37 und N 51 .

18 Bundesgesetz über den Schutz von Marken und Herkunftsangaben vom 28. August 1992 (Markenschutzgesetz, MSchG; SR 232.11).

19 Art.15 Abs.1MSchG; GLÖCKNER (Fn.7), S. 279.

20 Vgl. grundlegend BSK-HILTY, Art.1 UWG N 175: «[E]rst recht kommt das Gericht nicht umhin, ggf. ein Verhalten in Anwendung des UWG letztlich genauso zu verbieten, wie es dies z.B. in Anwendung des Urheberrechts, des Markenrechts oder des Designrechts tun müsste», m.H. auf ERIC PAHUD, Zur Kritik an der Umwegtheorie, sic! 2004, S. $808 \mathrm{f}$.

21 BAUDENBACHER (Fn. 9), Art. 2 N192; Heizmann/Loacker-HEINEMANN, $\S 5$ N1Fn.1.

22 Siehe stattdessen zum Verhältnis zwischen Kartellrecht und Recht des geistigen Eigentums vor allem ANDREAS HEINEMANN, Immaterialgüterschutz in der Wettbewerbsordnung - Eine grundlagenorientierte Untersuchung zum Kartellrecht des geistigen Eigentums, Tübingen 2002, S. 1 ff.; zum Verhältnis zwischen UWG und Immaterialgüterrechten FLORENT THOUVENIN, Funktionale Systematisierung von Wettbewerbsrecht (UWG) und Immaterialgüterrechten, Köln 2006, S. 503ff.; vgl. ferner Heizmann/Loacker-HEINEMANN, §5.

\section{Reduktion der Umwegthese auf ihre Kernanliegen}

Die Umwegthese besagt, dass qua Lauterkeitsrecht nicht 17 verboten werden kann, was qua Immaterialgüterrecht zulässig ist. ${ }^{23}$ Mit anderen Worten soll das UWG keinen (Leistungs-)Schutz bieten, wenn diesen schon das einschlägige Immaterialgüterrecht nicht gewährt. Damit spricht die Umwegthese eine der beiden zentralen Fragen des Verhältnisses zwischen Immaterialgüter- und Lauterkeitsrecht an. ${ }^{24}$ Auszugehen ist dabei von der treffenden Überlegung von PAHUD, wonach die Gesetze des Immaterialgüterrechts nicht nur positiv bestimmen, was sie schützen, sondern auch negativ, was sie nicht schützen, was also frei und anderen zugänglich sein soll. ${ }^{25}$

Das Bundesgericht beurteilte das Verhältnis zwischen 18 den beiden Rechtsgebieten in seiner älteren Rechtsprechung zunächst mittels Umwegthese wie folgt: Vor Inkrafttreten des UWG wurde mit dem lex specialis-Charakter des Markenschutzgesetzes (um nur einmal beim praxisrelevantesten Immaterialgütergesetz anzuknüpfen) argumentiert. Nach Inkrafttreten des ersten UWG von 1943 wurde auf die Notwendigkeit der Verhinderung von fraus legis (Gesetzesumgehung) verwiesen. ${ }^{26}$ Inzwischen lehnt das Bundesgericht die Umwegthese insofern ab, als es erklärt, die Normen des UWG seien im Verhältnis zum Markenschutz nicht subsidiär, sondern wiesen einen eigenständigen Anwendungsbereich auf. ${ }^{27}$

Nun soll in diesem Beitrag keineswegs einer Renaissance 19 der Umwegthese das Wort geredet werden. Dies umso weniger, als das UWG bekanntlich ohnehin keine erga omnes wirkenden Ausschliesslichkeitsrechte zu begründen vermag, wie sie die Immaterialgüterrechte darstellen. ${ }^{28}$ Zudem zeigt namentlich HEINEMANN völlig zu Recht auf, dass der Umgehungsgedanke schon wegen der

23 Vgl. Urteil des Bundesgerichts 4A_86/2009 vom 26. Mai 2009 E. 4 (nicht abgedruckt in BGE135 III 446).

24 Die andere zentrale Relationsfrage ist diejenige nach der Einschränkung des Immaterialgüterschutzes durch das Lauterkeitsrecht, vgl. Heizmann/Loacker-HEINEMANN, § 5 N 24; vgl. für das Kartellrecht Art. 3 Abs. 2 Satz1 KG.

25 PAHUd (Fn. 20), S. 806.

26 Heizmann/Loacker-HEINEMANN, §5 N29, m.H., insb. aufBGE 73 II 110 E. 4, und auch auf BGE 116 II 471 E. 3: «Die Nachahmung ist vielmehr grundsätzlich erlaubt, soweit nicht patent-, urheber- oder modellrechtliche Schutzansprüche entgegenstehen. Es ginge nicht an, auf dem Umweg über das Wettbewerbsrecht als widerrechtlich zu bezeichnen, was nach den Spezialgesetzen des gewerblichen Rechtsschutzes erlaubt ist», m.w.N.; vgl. BSK-HILTY, Art. 1 UWG N 160; MARBACH/DUCREY/WILD (Fn.13), N1154.

27 Urteil des Bundesgerichts 4A_86/2009 vom 26. Mai 2009 E. 4.1 und die dortigen weiteren Erläuterungen (nicht abgedruckt in BGE135 III 446).

28 MAGDA STREULI-YOUSSEF, in: STREULI-YOUSSEF (Hrsg.), Lauterkeitsrecht, Band V/1, 3. Aufl., Basel 2020, Art.3UWG N166; PAHUD (Fn. 20), S. $804 \mathrm{f}$. 
Inkommensurabilität von Lauterkeits- und Immaterialgüterrecht für die Verhältnisbestimmung wenig taugt. ${ }^{29}$ Im Ausgangspunkt wollte die Umgehungsthese aber $\mathrm{Wi}$ dersprüche zwischen Immaterialgüterrechten und Lauterkeitsrecht verhindern, indem das UWG die immaterialgüterrechtlichen Schutzrechtswertungen nicht durch weitgehende Annahme von Unlauterkeit konterkarieren sollte. ${ }^{30}$ Im Sinne der Einheit der Rechtsordnung hat dieses Anliegen selbstredend nach wie vor seine Berechtigung, weshalb die eng verwandten Materien des Immaterialgüterrechts und des Lauterkeitsrechts nicht völlig losgelöst voneinander angewandt werden sollten. ${ }^{31}$

Vor diesem Hintergrund besteht zwar keine Veranlassung, die Umwegthese in ihrer ursprünglichen Konzeption «wiederzubeleben» - ihre Grundanliegen sind aber weiterhin legitim. ${ }^{32}$ In Sachverhalten mit Bezügen sowohl zum Immaterialgüterrecht als auch zum UWG spricht dementsprechend wenig für eine isolierte Betrachtung des jeweils anderen Rechtsgebiets. ${ }^{33}$ Jedenfalls theoretisch trägt dem auch die Praxis des Bundesgerichts Rechnung. ${ }^{34}$

21 Auf derselben Linie der Förderung des Einheitsgedankens liegt es, wenn im Rahmen von Art. 3 Abs. $2 \mathrm{KG}^{35}$ allein die Frage interessiert, inwiefern das Kartellrecht den Immaterialgüterschutz beschränken können soll. Die Problematik der Schutzeinschränkung tritt auch im Lauterkeitsrecht auf, weshalb insofern Kongruenz zum Kartellrecht besteht. ${ }^{36}$ Die Diskussion aber, inwiefern Wettbewerbsrecht i.e.S. über die Immaterialgüterrechte hinaus einzelfallweise weiteren (faktischen) Leistungsschutz bieten soll, wird für das Kartellrecht naturgemäss nicht geführt.

29 Heizmann/Loacker-HEINEMANN, \$5 N30f

30 Vgl. Heizmann/Loacker-HEINEMANN, §5N 47f.; vgl. auch BSK-HILTY, Art. 1 UWG N163.

31 Heizmann/Loacker-HEINEMANN, § 5 N 47; BSK-HILTY, Art. 1 UWG N173.

32 Vgl. Heizmann/Loacker-HEINEMANN, § 5 N 48, der die Nachahmung als irrelevant für die Annahme von Unlauterkeit ansehen will. Ob ein Verstoss gegen das UWG vorliege, solle nur davon abhängen, ob eine der anerkannten, die Unlauterkeit begründenden Kategorien, z.B. Herkunftstäuschung, Rufausbeutung oder hinterlistiges Verhalten, gegeben sei; BSK-HILTY, Art. 1 UWG N 173; PETER JUNG, in:Jung/ Spitz (Hrsg.), Bundesgesetz gegen den unlauteren Wettbewerb (UWG), 2. Aufl., Bern 2016 (zit. SHK-BEARBEITERIN), Art. 2 UWG N 96.

33 Vgl. PAHUD (Fn. 20), S. 805.

34 Urteil des Bundesgerichts 4A_86/2009 vom 26. Mai 2009 E. 4.1, m.N. (nicht abgedruckt in BGE135 III 446): «Immerhin hat das Bundesgericht in seiner neuesten Rechtsprechung hervorgehoben, dass es der Kohärenz der Rechtsordnung widerspräche, wenn die für ein Recht ausdrücklich definierten Grenzen mit einer zu weit gezogenen Definition des Schutzes für ein anderes Recht unterlaufen werden könnten».

35 Bundesgesetz über Kartelle und andere Wettbewerbsbeschränkungen vom 6. Oktober 1995 (Kartellgesetz, KG; SR 251).

36 Vgl. Fn. 24.
Im Bereich des Lauterkeitsrechts sollte hinsichtlich des- 22 selben Aspekts, also der Möglichkeit einer Schutzerweiterung, zumindest grösste Zurückhaltung geübt werden: Dies zunächst schon deshalb, weil das UWG spätestens seit der Totalrevision von $1986^{37}$ - ähnlich dem Kartellrecht - auch den funktionierenden Wettbewerb schützen soll und nicht mehr nur dem Mitbewerberschutz verpflichtet ist. Ein extensiv verstandenes Rufausbeutungsverbot führt jedoch (rein statisch betrachtet) nicht $\mathrm{zu}$ einem Plus, sondern zu einem Minus an Wettbewerb was wiederum naheliegender Weise kein Grundanliegen des UWG sein kann. Angezeigt ist daher eine MehrebenenBetrachtung, die den Wettbewerbsförderungsanliegen von KG und UWG Rechnung trägt, gleichzeitig aber nicht jene des Immaterialgüterrechts ausblendet. $\mathrm{Zu}$ denken ist insofern vor allem an Innovationsanreize durch Leistungsschutz und, im Markenschutzrecht, an Suchkostenverringerung, wobei die Generierung von Innovation, nach vorzugswürdigem dynamischem Verständnis des Wettbewerbs, ohnehin auch Intention des Wettbewerbsrechts ist. ${ }^{38}$ Wie weit die Innovationsanreize zu schützen sind, entscheidet aber grundsätzlich der Immaterialgüterrechtsgesetzgeber. ${ }^{39}$ Die immaterialgüterrechtlich abgesteckten Grenzen dürfen deshalb nur in besonderen Ausnahmefällen einer faktischen Erweiterung auf lauterkeitsrechtliche Grundlage zugeführt werden.

\section{Reduktion der «Rufausbeutung» auf die eindeutigsten Fälle}

Das Obige legt eine Zurückführung des Anwendungsbe- 23 reichs der UWG-widrigen Rufausbeutung auf die eindeutigsten Fälle nahe. ${ }^{40}$ Das sind jene, in denen die transparenzerhöhende Wirkung (Lenkungsfunktion) einer Rufausbeutung gering bleibt und gleichzeitig die mit der Rufausbeutung immer einhergehende Beeinträchtigung der Verteilungsfunktion nicht aufgewogen wird. ${ }^{41}$ Die hier befürwortete Mehrebenen-Betrachtunggebietet demnach nicht bloss die Respektierung der gesetzgeberischen Wertungen im Immaterialgüterrecht, sondern auch jener im UWG, namentlich dessen Anlegen der Sicherstellung der Funktionsfähigkeit des Wettbewerbs. ${ }^{42}$

Anders zu entscheiden bedeutete nicht nur, die Wertun- 24 gen des Immaterialgüterrechts zu unterlaufen, sondern

\section{Heizmann/Loacker-THIER, §2 N $21 \mathrm{ff}$.}

38 Vgl. ANDREAS HEINEMANN, Immaterialgüterrecht und Wettbewerbsrecht: Divergenz oder Konvergenz?, Medien und Recht - International Edition 2014, S. 6 (zit. MR-Int); BSK-HILTY, Art. 1 UWG N153 ff.

39 PAHUD (Fn. 20), S. 807.

40 Vgl. auch BGE135 III 446 E. 7.5: «Denn das Verbot der unlauteren Anlehnung an eine Konkurrenzausstattung soll nur eindeutige Fälle unnötiger Anlehnungen erfassen, die nicht durch ein Informationsbedürfnis zu rechtfertigen sind».

41 THOUVEnin (Fn. 22), S. 443 f.; vgl. GLÖCKNER (Fn. 7), S. 274 ff.

42 Dazu für viele Heizmann/Loacker-HEIzMANN, Art. 1 N $27 \mathrm{ff}$. 
auch, im Lauterkeitsrecht faktisch einer antiquierten Vorstellung des reinen Konkurrentenschutzes verhaftet zu bleiben. Gerade diese stand freilich noch am Anfang des Konzepts der Rufausbeutung: Entwickelt wurde es nämlich in einem bundesgerichtlichen Urteil von 1976 auf Grundlage der «alten» Generalklausel des UWG von $1943^{43}$, das bekanntlich - obgleich nicht konzeptionell, so doch in seiner Anwendung - dem Mitbewerberschutz und nicht dem Funktionsschutz des Wettbewerbs verpflichtet war ${ }^{44}$. Diese Rechtslage ist indessen heute offenkundig ganz anders akzentuiert. ${ }^{45}$

Angesichts dessen sollten die Anforderungen an eine erfolgreiche Berufung auf die Rufausbeutung hoch angesetzt werden. So ist jedenfalls vorauszusetzen, dass eine entsprechende Bekanntheit gegeben ist. Das Handelsgericht prüfte diesen Aspekt zwar, liess letztlich aber offen, ob «dieser Nachweis wirklich erforderlich ist». Gleichzeitig führte es aus, es sei «höchstens» Bekanntheit zu verlangen (E. 27.4).

26 Nach dem hier befürworteten Verständnis sollte exakt das Gegenteil gelten: Die Rufausbeutung verlangt «mindestens» die Bekanntheit des betreffenden Produkts, ${ }^{46}$ wenn nicht überhaupt dessen Verkehrsdurchsetzung. Nur durch eine solche Restriktion des Anwendungsbereiches wird der Gefahr einer allzu grosszügigen Eröffnung der Rechtsfigur der Rufausbeutung hinreichend begegnet. Vor demselben Hintergrund sollte nicht nur die Herabsetzung, sondern auch die erfolgte Anlehnung unnötig sein, damit der Tatbestand erfüllt ist. Der Gesetzeswortlaut steht dem zumindest nicht entgegen («in [...] unnötig herabsetzender oder anlehnender Weise»). ${ }^{47}$

27 Des Weiteren sollte in jedem Anwendungsfall sorgsam geprüft werden, wie es um die Wirkungen des fraglichen Verhaltens auf die Wettbewerbsordnung steht und welche grundlegenden Wertungsentscheide im Immaterialgüterrecht getroffen wurden. Auf diese Weise muss eine überzeugende Sachverhaltsbeurteilung unter Reflektion beider (Rechts-)Ebenen erfolgen. ${ }^{48}$ Im Regelfall dürfte die gesetzgeberische Abwägung im Immaterialgüterrecht zwischen Begrenzungsinteressen und den Interessen der

43 BGE102 II 292 E. 5.-7., Lattoflex; Botschaft vom 18. Mai $1983 \mathrm{zu}$ einem Bundesgesetz gegen den unlauteren Wettbewerb (UWG) (BBl1983II1009), S.1064f.

44 Heizmann/Loacker-HEIZMANN, Art. 1 N1f., m.N.

45 Dementsprechend überhaupt für ein exklusives Abstellen auf den funktionalen Schutzzweck THOUVENIN (Fn. 22), S.153f.

46 Die Ausbeutung des Rufs eines Produkts, das unbekannt ist, ist ohnehin tatsächlich kaum vorstellbar.

47 Vgl. nur Heizmann/Loacker-STAUBER/ISKIC, Art. 3 Abs. 1 lit.e, N 50 Fn.119, und Botschaft zum UWG (Fn. 43), S. 1064.

48 Vgl. ferner BGE129 III 353 E.3.3, wonach auf der anderen Seite der Inhaber einer Marke diese nicht unlauter verwenden darf; vgl. auch GLÖCKNER (Fn.7), S. 274, m.w.N.
Allgemeinheit massgeblich bleiben. ${ }^{49}$ Jedenfalls ist wie schon im Immaterialgüterrecht eine Overprotection ${ }^{50} \mathrm{der}$ Leistungen auch im Bereich des Lauterkeitsrecht unbedingt zu vermeiden.

Am gegenständlichen Entscheid des Handelsgerichts 28 ist zu kritisieren, dass er die Prüfung des Verhältnisses zum Immaterialgüterrecht unterliess oder zumindest nicht ersichtlich machte. Dass sich das Handelsgericht dieser Problematik nicht bewusst gewesen sein könnte (E. 27.3) ${ }^{51}$, legt auch dessen unbesehene Bezugnahme auf BGE 126 III 315 (Rivella/Apiella) (E. 28.7) nahe. Denn dieser Entscheid erging vornehmlich gestützt auf das Markenschutzgeset $\mathrm{z}^{52}$ und nur hilfsweise unter Bezugnahme auf Art. 3 Abs. 1 lit. d UWG53 - während Art. 3 Abs. 1 lit. e UWG überhaupt keinen Gegenstand der Überlegungen bildete.

Abgesehen davon war der vom Handelsgericht in Bezug 29 genommene Leitentscheid des Bundesgerichts zur Rufausbeutung aus dem Jahr 1976 anders gelagert als der vorliegende des Berner Gerichts: So hatte die Beklagte nicht nur ihre «Bico-flex»-Untermatratze bewusst als Gegenprodukt zur «Lattoflex»-Untermatratze etabliert, sondern sie wies in einem eigenen Schreiben explizit darauf hin, dass ihr Produkt der Lattoflex-Matratze sogar überlegen und trotzdem um mehr als $20 \%$ billiger sei. Dies legte die Absicht nahe, den guten Ruf eines Mitbewerbers und seines auf dem Markt eingeführten Erzeugnisses der eigenen Werbung dienstbar zu machen. ${ }^{54}$

Im jüngsten Berner Fall erfolgte die Rufausnutzung hin- 30 gegen gerade nicht «offen», sondern allenfalls verdeckt, nämlich anhand des Produktnamens und mit einem Teil der Ingredienzen. Die äussere Warenform hingegen war deutlich verschieden gestaltet ${ }^{55}$, zudem handelt es sich beim «Ingwerer» um ein Shot-Getränk, während der «Summerer» als Mischgetränk konzipiert war (E. 28.3), und in der Werbung pries die Beklagte ihr Produkt namentlich als Zitronen-, nicht aber als Ingwer-Likör an ${ }^{56.57}$ Werbeschreiben o.ä. wurden nicht bekannt. Eine unmittelbare Verwechslungsgefahr dürfte nicht bestanden haben.

49 PAHUD (Fn. 20), S. 807.

50 HEINEMANN, MR-Int (Fn. 38), S. 6.

51 Vgl. aber immerhin das Handelsgericht, dass auf den Beitrag von GLÖCKNER verweist (vgl. vorstehende Fn. 7).

52 BGE126III315E. 6.

53 Die kurzen Erwägungen dazu wurden denn auch nicht publiziert, BGE126 III 315 unpubl. E. 7 (vgl. Urteil des Bundesgerichts 4C.316/1999 vom 18. Juli 2000).

54 BGE102 II 292 E. 7 .

55 Vgl. die Abbildung in Der Bund vom 24. Februar 2020 («Ingwerer gewinnt Streit der Berner Likörhersteller»).

56 Bärnerbär vom 2.Juli 2019 («Ein Zitronen-Likör, der den Sommer in der Flasche festhält»).

57 Vgl. auch die Argumente der Beklagten (E. 28.3). 

nähere Aktenkenntnisse schwierig ist, hätten die vorstehenden Überlegungen insgesamt wohl eher dafür gesprochen, den «Summerer» in Wettbewerb mit dem «Ingwerer» treten zu lassen. Dieses Ergebnis hätte sich überdies mit der Wertung in BGE135 III 446 gedeckt, in welchem eine Rufausbeutung in Bezug auf eine anlehnende Ausstattung abgelehnt wurde («Maltesers»).

Folgt man dem, hätten in Vermeidung einer lauterkeitsrechtlichen Overprotection faktischer Ausschliesslichkeitsinteressen die Kundenpräferenzen über das weitere Schicksal der Produkte entscheiden sollen und nicht das Handelsgericht. Dazu kann es nun indessen nicht mehr kommen, denn die Beklagte befindet sich spätestens seit der Ausfertigung des Entscheids am 9.Juni 2020 in Liquidation. 58

\section{Resümee und Ausblick}

Fälle wie jener, den das Berner Handelsgericht zu beurtei len hatte, sind auch in rechtsvergleichender Umschau ${ }^{59}$ keineswegs selten. Sie verdienen daher insbesondere mit Blick auf absehbare Folge-Entscheidungen sorgsamer Analyse. In besonderem Mass gilt dies hinsichtlich des seit jeher in den Details umstrittenen Verhältnisses von Lauterkeits- und Markenrecht. Ungeachtet aller Unterschiede ist nicht zu verkennen, dass beide Rechtsgebiete eine parallele Stossrichtung mit Blick auf den Funktionsschutz des Wettbewerbs verfolgen können; ${ }^{60}$ für das Markenrecht ist dies gegenüber anderen Immaterialgüterrechten besonders offensichtlich. ${ }^{61}$ Hinzu kommt (wie der hier besprochene Anlassfall dokumentiert) die bisweilen praktische Gleichwertigkeit des einmal erwirkten Rechtsschutzes ${ }^{62}$ - sei dieser nun (gleichsam: verdeckt) lauterkeitsrechtlich oder (offen) immaterialgüterrechtlich gewährt worden.

58 Was offenbar zugleich zur Folge hatte, dass die Klägerin die Gerichts- und Anwaltskosten zu tragen hatte, Der Bund vom 22.Juli 2020 ("Ingwerer-Konkurrentin ist konkurs»).

59 Vgl. etwa schon den Entscheid des österreichischen Obersten Gerichtshofs vom 29. Oktober 1996, 4 Ob 2200/96 = ÖBl 1997, 72 bei dem ein Hersteller von Wurstwaren durch Hervorhebung des Wortes «Schürzenjäger» im Produktnamen vom Image einer (jedenfalls vormals) sehr bekannten, gleichnamigen Band zu partizipieren versuchte. Aus dem Bereich des deutschen Rechts erlangte früh das Urteil des Bundesgerichtshofs vom 9. Dezember 1982, IZR133/80 = GRUR 1983, 247 grössere Bekanntheit, in dessen Ausgangssachverhalt ein Whiskey-Hersteller durch anzeigenmässige Abbildung seines Produkts vor einem englischen Luxusauto etwas von dessen Noblesse einfangen wollte.

60 Siehefür das deutsche Recht nur WOLFGANG B. SCHÜNEMANN/KARLNIKOLAUS PEIFER, in: Peifer (Hrsg.), UWG, Band1, 3. Aufl., Berlin 2020, Einleitung, S. 619 .

61 Vgl. (wiederum aus deutscher Perspektive) SCHÜNEMANN/PEIFER (Fn.60), Einleitung, S.624.

62 Vgl. Rz.15.
Insgesamt ist mit dem Bundesgericht ${ }^{63}$ zwar von einer 34 gänzlichen Eigenständigkeit (d.h. Gleichrangigkeit und nicht etwa einem schlichten Vorrang-Nachrang-Verhältnis) beider Rechtsbereiche auszugehen. Allerdings ist diese Eigenständigkeit keineswegs mit einer Art «allgemeiner Auffangfunktion» des UWG für Durchsetzungsdefizite oder -schwierigkeiten von Immaterialgüterrechten gleichzusetzen. Mag auch aus heutiger Sicht die vormalige Handhabung und Ausprägung der sog. Umwegthese ${ }^{64}$ durch das Bundesgericht gewiss überholt sein, ein Anliegen suchte sie jedenfalls zu Recht zu verwirklichen, nämlich die Vermeidung von Wertungswidersprüchen. Nichts anderes zeichnet die insofern bemerkenswert parallele Rechtsentwicklung in Deutschland ${ }^{65}$ und Österreich ${ }^{66}$ aus. Angesichts ihrer überkommenen methodischen Prämissen ${ }^{67}$ könnte man die vormalige bundesgerichtliche Umwegthese im Rückblick auch als einen Umweg zu einem legitimen Ziel, konkret jenem der Wahrung der Einheit der Rechtsordnung ${ }^{68}$ charakterisieren.

Was sich heute deshalb u.E. als echter Ausweg anbietet, 35 ist eine Mehrebenen-Betrachtung, die weder qua UWG leichtfertig "ergänzt», ${ }^{69}$ was im Immaterialgüterrecht mit guten Gründen anders abgesteckt wurde noch sich per se jedwedem Schutz qua UWG versperrt, nur weil Berührungspunkte namentlich zum Markenrecht bestehen. Vermieden werden soll so insbesondere die Entstehung von unbefriedigenden Zielkonflikten, die einheitliche Lebenssachverhalte für die beiden unterschiedlichen Rechtsbereiche bergen können. Auf diese Weise gesellt sich zur Betonung der Eigenständigkeit ein weiteres Element der beachtungswürdigen Wechselwirkungen zwischen den beiden Materien hinzu.

Im vorliegend konkret diskutierten Kontext wird der pro- 36 pagierten Mehrebenen-Betrachtung am ehesten durch ein restriktives Verständnis der Rufausbeutung Genüge getan, da anderenfalls lauterkeitsrechtlich herbeigeführt wird, was markenrechtlich als Overprotection verhinderungswürdig ist. Dementsprechend wird man zumindest

\footnotetext{
63 Siehe zuvor Rz.18 a.E.

64 Zuihr Rz.17.

65 Vgl. instruktiv HELMUT KöHLER, in: Köhler/Bornkamm/Feddersen (Hrsg.), Gesetz gegen den unlauteren Wettbewerb, 39. Aufl., München 2021, §4 UWG N3.6f.

66 Siehe nur MATHIAS GÖRG, Kommentar zum UWG, Wien 2020, §1 UWG N1233 ff.

67 Siehe Rz.19ff.

$68 \mathrm{Zu}$ diesem Fundamentalprinzip wegweisend (abgesehen von teils schauerlichem, dem damaligen «Zeitgeist» geschuldeten Vokabular - vgl. etwa die dortige S. 9) KARL ENGISCH, Die Einheit der Rechtsordnung, Darmstadt 1935 (zuletzt Nachdruck 1987) sowiejüngst den Sammelband von PHILIPP HELLWEGE / MARTA SONIEWICKA, Die Einheit der Rechtsordnung: Annäherungen, Bestandsaufnahmen, Reflexionen, Tübingen 2020.
}

69 Entsprechend kritisch zum Verständnis eines ergänzenden UWGLeistungsschutzes denn auch explizit KöHLER (Fn. 65), §4UWG N3.6. 
den Nachweis hinreichender Bekanntheit, eher aber noch eine entsprechende Verkehrsdurchsetzung (hier: des deshalb schutzwürdigen Produkts) voraussetzen müssen. ${ }^{70}$ Alles andere heisst, immaterialgüterrechtlichen Schutz in das UWG vorzuverlagern bzw. auszulagern, obwohl weder der Funktionsschutz des Wettbewerbs noch das Immaterialgüterrecht selbst dazu Veranlassung geben. Gleichrangigkeit von Rechtsquellen bedeutet eben immer auch eine besondere Begründungslast bei der Befürwortung von ausnahmsweisen Durchbrechungen.

70 Vgl. dazu Rz. 26.
Wird dem nicht hinreichend Rechnung getragen, droht nicht nur ein Konterkarieren immaterialgüterrechtlicher Wertungen, sondern last, not least auch eine problematische Verengung legitimer wettbewerblicher Verhaltensspielräume. ${ }^{71}$ In eben dieser Hinsicht bot das hier gegenständliche Urteil des Handelsgerichts Bern nicht nur Anlass zu einigen ergänzenden, grundlegend-systematischen Überlegungen, sondern vor allem Angriffsfläche hinsichtlich des mit ihm erzielten Ergebnisses.

71 So zum zuletzt genannten Aspekt explizit OLAF SOSNITZA, in: Heermann/Schlingloff (Hrsg.), Münchener Kommentar zum Lauterkeitsrecht, Band1, 3. Aufl., München 2020, §3 N92. 